\title{
Development of an Integrative Care Program in a Pediatric Oncology Unit
}

\author{
Britta Rutert $^{\mathrm{a}} \quad$ Wiebke Stritter $^{\mathrm{b}} \quad$ Angelika Eggert $^{\mathrm{b}} \quad$ Ulrike Auge $^{\mathrm{b}}$ \\ Alfred Laenglerc Georg Seifert ${ }^{b, d}$ Christine Holmberg ${ }^{e, f}$ \\ ${ }^{a}$ Berlin-Brandenburg Academy of the Sciences and Humanities, Berlin, Germany; ${ }^{b}$ Department of Paediatric \\ Oncology/Haematology, Otto-Heubner Centre for Paediatric and Adolescent Medicine (OHC), Charité - \\ Universitätsmedizin Berlin, Berlin, Germany; ${ }^{c}$ Division of Oncology and Hematology, Institute for Pediatrics, \\ Gemeinschaftskrankenhaus Herdecke, Witten-Herdecke University, Witten-Herdecke, Germany; ${ }^{\mathrm{d}}$ Institute of Social \\ Medicine and Epidemiology, Brandenburg Medical School Theodor Fontane, Brandenburg, Germany; ${ }^{\mathrm{e}}$ Faculty of \\ Health Sciences Brandenburg, Brandenburg Medical School Theodor Fontane, Potsdam, Germany; 'Departamento \\ de Pediatria, Faculty of Medicine, University of São Paulo, São Paulo, Brazil
}

\section{Keywords}

Integrative care · Oncology · Pediatrics · Program

development

\begin{abstract}
Purpose: The aim of this article is to describe what needs to be considered in implementing care practices, in this case an integrative care program consisting of anthroposophic treatments, in an intensive care unit (ICU) of a teaching hospital. Methods: We used a pediatric oncology department to implement an integrative care program. We conducted a qualitative study including participant observation and semi-structured interviews with parents, nurses, doctors, and therapists. Data analysis was based on a grounded theory approach and focused on the status quo of care in the ICU. Results: The following factors needed to be considered: the structure of the ICU, communication and information dissemination, and time constraints. This led to the following components of the integrative care program: (1) a training plan in anthroposophic treatments for all nurses that was conducted by 2 trained anthroposophic nurses, and (2) the introduction of an integrative shift that was on top of regular
\end{abstract}

Georg Seifert and Christine Holmberg contributed equally to this work.

karger@karger.com

www.karger.com $/ \mathrm{cmr}$

Karger $\%$

GOPEN ACCESS
2020 The Author(s)

Published by S. Karger AG, Basel

This is an Open Access article licensed under the Creative Common Attribution-NonCommercial-4.0 International License (CC BY-NC) (http://www.karger.com/Services/OpenAccessLicense), applicable to the online version of the article only. Usage and distribution for commercial purposes requires written permission. care at the ICU and focused on delivery of integrative care to patients. Conclusion: To add new care components to an $\mathrm{ICU}$, the existing context has to be considered. Time constraints and high workload are factors that need to be recognized. In this particular context, a highly flexible program was the solution.

(c) 2020 The Author(s)

Published by S. Karger AG, Basel

\section{Entwicklung eines Integrativen Pflegeprogramms auf einer kinderonkologischen Station}

\section{Schlüsselwörter}

Integrative Pflege · Onkologie · Pädiatrie .

Programmentwicklung

\section{Zusammenfassung}

Ziel: Ziel dieses Artikels ist es zu beschreiben, welche Bedingungen für die Implementierung eines Integrativen Pflegeprogramms auf einer kinderonkologischen Intensivstation einer Universitätsklinik notwendig sind. Methoden: Auf einer kinderonkologischen Station wurde ein Integratives Pflegeprogramm implementiert. Zur Entwicklung des Programms wurde eine qualitative Studie mit teilnehmender Beobachtung und halb-strukturierten In- 
terviews mit Eltern, Krankenschwestern, Ärzten und Therapeuten durchgeführt. Die Datenanalyse erfolgte mit Hilfe von Grounded Theory und konzentrierte sich auf die Analyse des Status quo der Station. Ergebnisse: Die folgenden Faktoren wurden bei der Entwicklung des Programms berücksichtigt: die Struktur der Intensivstation, Kommunikation und Informationsverbreitung, sowie zeitliche Einschränkungen. Daraus resultierten die folgenden Komponenten des Integrativen Pflegeprogramms: (1) ein Trainingsplan zur Vermittlung von pflegerischen Anwendungen der anthroposophischen Medizin für alle Krankenschwestern, welcher von zwei in anthroposophischer Pflege ausgebildeten Schwestern trainiert wurde, und (2) die Einführung einer Integrativen Schicht, die zusätzlich zu dem regulären Schichtensystem eingeführt wurde, um integrative Pflege durchzuführen. Schlussfolgerung: Folgende Kontextfaktoren sollten bei der Einführung eines Integrativen Pflegeprogramms in einem Universitätsklinikum mit einbezogen werden: zeitliche Einschränkungen und starke Arbeitsbelastung des Pflegeteams. Auf der hier untersuchten kinderonkologischen Station war die Einführung eines flexiblen Programms die Lösung.

(c) 2020 The Author(s) Published by S. Karger AG, Basel

\section{Introduction}

Treatment for children with cancer has improved significantly over the last decades, and the overall survival rate of cancer in children today is higher than $80 \%$ [1]. Such treatments have considerable side effects, such as pain, nausea, or anxiety. These side effects are commonly treated with conventional medicine, such as painkillers, antibiotics, or sedatives [2], even though some side effects could be relieved with components of traditional and complementary medicine (T\&CM). The general use of T\&CM is widespread in the population, especially among cancer patients [3]. Research has shown that in Germany $72 \%$ of all patients have made use of T\&CM [4], and a survey revealed that $35 \%$ of children with cancer were treated with or used T\&CM [5-7]. In the United States, $40 \%$ of cancer patients used T\&CM, and doctors have reported making use of T\&CM [8] and merging the 2 systems of T\&CM and conventional medicine. This trend is known under the term "integrative medicine" (IM). IM and care refer to the interdisciplinary blending of conventional medicine and care, and T\&CM with the purpose of enhancing patients' health [9-11]. It can thus also be summarized as complementary and integrative medicine (CIM). It is "supportive care rather than a replacement of conventional medical care" [12] and may be leading to "synergistic therapeutic effects" [13]. While T\&CM/CIM is perhaps more psychologi- cally accessible to many patients in that it better reflects commonly held values, it is often less financially and institutionally accessible [14]. Nevertheless, different models to integrate T\&CM modalities into conventional medicine and care have been developed since the 1990s [15], and some hospitals and clinics in Europe and the USA have begun to offer T\&CM modalities [16, 17]; these include acupuncture, chiropractic, yoga, massage therapy, naturopathy, and anthroposophic medicine [5]. While IM is slowly finding its way into the German hospital landscape [18], university hospitals, with their focus on high-technology medicine and academic research, still have an ambivalent or even denunciatory attitude to T\&CM/CIM [19]. Although openness towards T\&CM/CIM is prevailing, one reason for difficulties in merging the 2 domains lies in what Pérard et al. [20] define as major cultural differences between conventional and traditional, complementary, or integrative medicine and their differing philosophies, historical development, and settings.

This article describes the development of a program that intends to complement integrative care with conventional care in a pediatric oncology intensive care unit (ICU) of a university hospital in Germany. Instead of copying already existing models of other clinics, this program is a new approach that looks at and includes the specific conditions and structures of the ICU. The program presented here is part of a larger 3-year research project, which aims to develop, implement, and evaluate integrative care as an integral and regular part of care in the pediatric oncology ICU [21]. In integrative care, the nursing repertoire is broadened by additional use of interventions, such as compresses or rhythmic embrocation [22]. The aim of this is to bring the positive effects of touch and affection into daily care [23-26], which often falls short in the busy and tight working schedule of nurses [27]. The integrative care program described in this article is based on anthroposophic treatments, in this case, rhythmic embrocation and oil compresses. Our ICU is one of a few pediatric cancer treatment centers in Germany. It offers space for 19 young patients, who receive chemotherapy for all types of cancer. Treatment in the ICU is based on the highest standards of conventional medicine and care. Care is provided by 29 nursing staff members in full- and part-time positions. So far, no T\&CM modalities have been applied in the ICU. The central questions in the development of the program were thus: How can anthroposophic treatments be integrated in the specific structure and conditions of the pediatric oncology ICU? How can conventional care and integrative care be merged under the specific conditions? 


\section{Methods}

To develop the integrative care program, we conducted a qualitative study with several components: participant observations in the ICU and at 2 different anthroposophic hospitals, and semistructured interviews with health personnel at the anthroposophic hospitals. Data collection lasted 7.5 months between September 2016 and August 2017.

\section{Data Collection}

One week of participant observation and semi-structured interviews with health personnel (doctors, therapists, and nurses) was conducted at an anthroposophic hospital on a pediatric ward offering oncological treatment in order to understand integrative care in a hospital setting. Another week of participant observation on an oncology ward of a second anthroposophic hospital and semi-structured interviews with health personnel (doctors, therapists, and nurses) was conducted in order to understand integrative care in yet another setting.

A month of participant observation was conducted in the pediatric oncology ICU in order to investigate the status quo of the ward. The observation focused on daily interactions and communication structures between patients and nurses as well as the organization of care on the ward. These phases were followed by a phase of data analysis to understand how a training curriculum to teach all nurses in anthroposophic treatments could best be applied in the ICU. A training curriculum was developed and put into effect in the ICU. The implementation of the training phase lasted 6 months and was followed by participant observation looking at the interaction and communication between the trainers and the nurses during the training and the structures on the ward during the training phase as well as the acceptance of anthroposophic treatment.

\section{Data Analysis}

Interviews were transcribed verbatim and field notes were written of the participant observations. All written materials including protocols of the interviewers were entered into MAXQDA ${ }^{\circledR}$ (qualitative analysis software). Analysis was based on a grounded theory approach $[28,29]$. In a first step, initial interviews and field notes were coded in their entirety and codes were developed from the materials. This open inductive coding process was followed by a nuanced analysis with a particular focus on the research question. This was done by re-reading and re-coding the material and integrating it finally into core categories. This more nuanced analysis and coding was performed to guide the development of the integrative care concept. The core research team consisted of a social anthropologist (B.R.) with a long history of ethnographic research and data analysis and a social anthropologist and psychologist (U.A.) with extended knowledge in grounded theory. Crosschecking the data analysis process helped prevent biased perception of the data. Regular data meetings with an extended team of researchers added to the reliability of data [30].

\section{Results}

During the period of 7.5 months of participant observation on the different wards and in different phases of program development, 44 observational protocols were completed, 20 during the status quo assessment and 24 during the training period. Altogether, 12 semi-struc- tured interviews with nurses, therapists, and doctors were completed at 2 different anthroposophic hospitals. Data coding and analysis of the observational records and interviews, ongoing presence and observation in the ICU, and regular communication with all relevant stakeholders yielded 3 major categories, which were considered in the development of the integrative care program: (1) the structure of the ward, (2) communication and information dissemination on the ward, and (3) time. In the following sections, we show the relevance of these categories and their impact on the development of the training and care program.

\section{Structure of the ICU}

Care in the ICU is organized in a 3-shift system with morning, afternoon, and night shifts. All shifts are highly labor intensive, with a labor peak in the morning shift. The morning shift ends with the handover of patient information from the morning to the afternoon shift at 2 p.m. The official numbers of nurses working in this ICU are 5 nurses in the morning shift, 4 nurses in the afternoon shift, and 3 nurses in the night shift. Shifts were, however, often under-staffed due to the shortage of nurses, who often changed due to pregnancies, sick leave, and retirement. Generally, the ward was subject to frequent fluctuations in nurses and patients. Patients stayed from 1 day to several weeks, depending on the treatment plan. The many changes resulted in a partially unstable structure on the ward and an atmosphere of tension, especially when the shifts could not be manned completely. An experienced and highly motivated core nursing team that filled existing staff gaps compensated during this ongoing challenge.

\section{Communication and Information Dissemination}

The ICU is a place of ongoing and lively communication and interaction between patients, parents, nurses, and doctors. Information on nursing-related issues was disseminated in the monthly nursing team meeting. Among the nurses, daily information on the patients was passed on in the handover at 2 p.m. Information between nurses and doctors was exchanged in the ward round in the morning shifts. The absence of an overall multi-professional team meeting led to occasional information gaps or misunderstandings between the different professions. This was an often-repeated subject of complaint in the nursing team. In addition, communication between parents, patients, and nurses was often dependent on the nurses' time, and nurses often complained about the lack of time for appropriate communication with parents and care for patients. The need for more communication was a constant subject on the ward and important information for the conceptualization of the integrative care model. 
Time

Apart from the lack of communication, the lack of time was a constant issue on the ward. The work with children is time consuming, since children often need more attention than adult patients do. Basic care, such as taking vital signs and distributing medication, clinical examination and administration, absorbs a lot of the available time, especially in the morning shift. It is even more time consuming when the shift is under-staffed, which was a constant subject of complaint in the nursing team. Afternoon shifts were mostly quieter. Especially the time between 5 p.m. and 7 p.m. was quiet, as clinical examinations and ward rounds had been done earlier and supper had not yet been served. Therefore, time for additional activities and communication was available, which included child-friendly activities, such as watching movies or cooking with the children.

The 3 aspects structure, communication and information, and time are strongly interlinked. The lack of time and tight structures during the shifts led to a lack of communication, which led to complaints in the nursing team of not having enough time for the patients. Even though time and communication are a scarce resource in the ICU, the nursing team in cooperation with the head of the ward decided to implement integrative care in the ICU. This was also motivated by the wish to bring time, communication, as well as touch and affection, back into care. The care program was thus developed along the abovementioned determinants.

\section{The Integrative Care Program}

The specific structure of the ward (e.g., handover from morning to afternoon shift at 2 p.m.) produced a defined period in which a training curriculum to train all nurses in anthroposophic treatments could be included. First, the training curriculum to train the nurses in the treatments was developed and initiated. Before the actual training, 2 expert nurses from an anthroposophic training center were asked to attend the ward during morning and afternoon shifts to identify the best time for the training.

Training Curriculum

Pre-Training Phase. For a period of 1 month, the 2 expert nurses attended morning and afternoon shifts to identify the best time for the training. As observed before, morning shifts were busy with examinations, ward rounds, and basic care. Afternoon shifts were considerably quieter. To receive information on the patients, attending the shift handover at 2 p.m. was vital. Knowing the patients helped decide which anthroposophic treatments they should receive. Thus, the time between 2 p.m. and 6 p.m. proved to be the best for the actual training.
Training Phase. For a period of 5 months, the 2 expert nurses then trained the nurses of the ICU in the preparation and application of oil compresses and in rhythmic embrocation of the feet, arms, belly, and back, depending on the patients' indications. To really reach all nurses, the expert nurses also attended 2 nursing team meetings to present the treatments. To integrate the treatments into daily care, the 2 expert nurses went through every patient room to offer the treatments. To meet the structural and temporal constraints of the ICU, specific simplified versions of rhythmic embrocation and indication-based oil compresses were developed in the course of the training. In addition, a list of the most prevalent indications (e.g., nausea, anxiety, or constipation) and relevant oils was developed and filed, together with instructions on the application of compresses and embrocation, in a folder available to all nurses on the ward. All material and folders were stored in a cabinet in the ICU. In addition, a team of 5 nurses was elected from the overall nursing team to receive extra training in anthroposophic treatment at a school for anthroposophic care outside the university hospital. The core team of 5 nurses was chosen to apply the integrative care model and train other nurses and parents in the anthroposophic treatment. As data revealed, the long-term presence of the 2 expert nurses on the ward in addition to the core team led to a change (from initial resistance) to acceptance of the treatments on the ward. Initial resistance expressed as "I want to do it, but I don't have the time" changed to acceptance of the new care modalities. In addition, training of parents and of every nurse on the ward contributed further to the change in attitude. Therefore, the length of time necessary and trust in staff are 2 central qualities in the training development.

\section{The Program in the ICU}

Based on the results of the preceding data analysis, the implementation and documentation of the training, ongoing communication with the relevant stakeholders, as well as the structural conditions of the ward, an integrative care program was developed. This is applicable to the structure of the ward and will be implemented after the development phase. The basis of this program is an integrative shift in which standard and flexible integrative care will be provided. One of the 5 specifically trained nurses (integrative nurses) will execute the integrative shift in a rotating system.

Integrative Shift. The integrative shift consists of anthroposophic treatments offered 3 times a week from 12 p.m. to 8 p.m. ( 8 h due to the overall working time framework of the ward). In these $8 \mathrm{~h}$, the integrative nurse, 1 of 5 rotating nurses specifically trained in anthroposophic treatments, will go through every patient room to identify the needs of patients, prepare and apply the respective treatments, teach parents to apply these treatments, and 


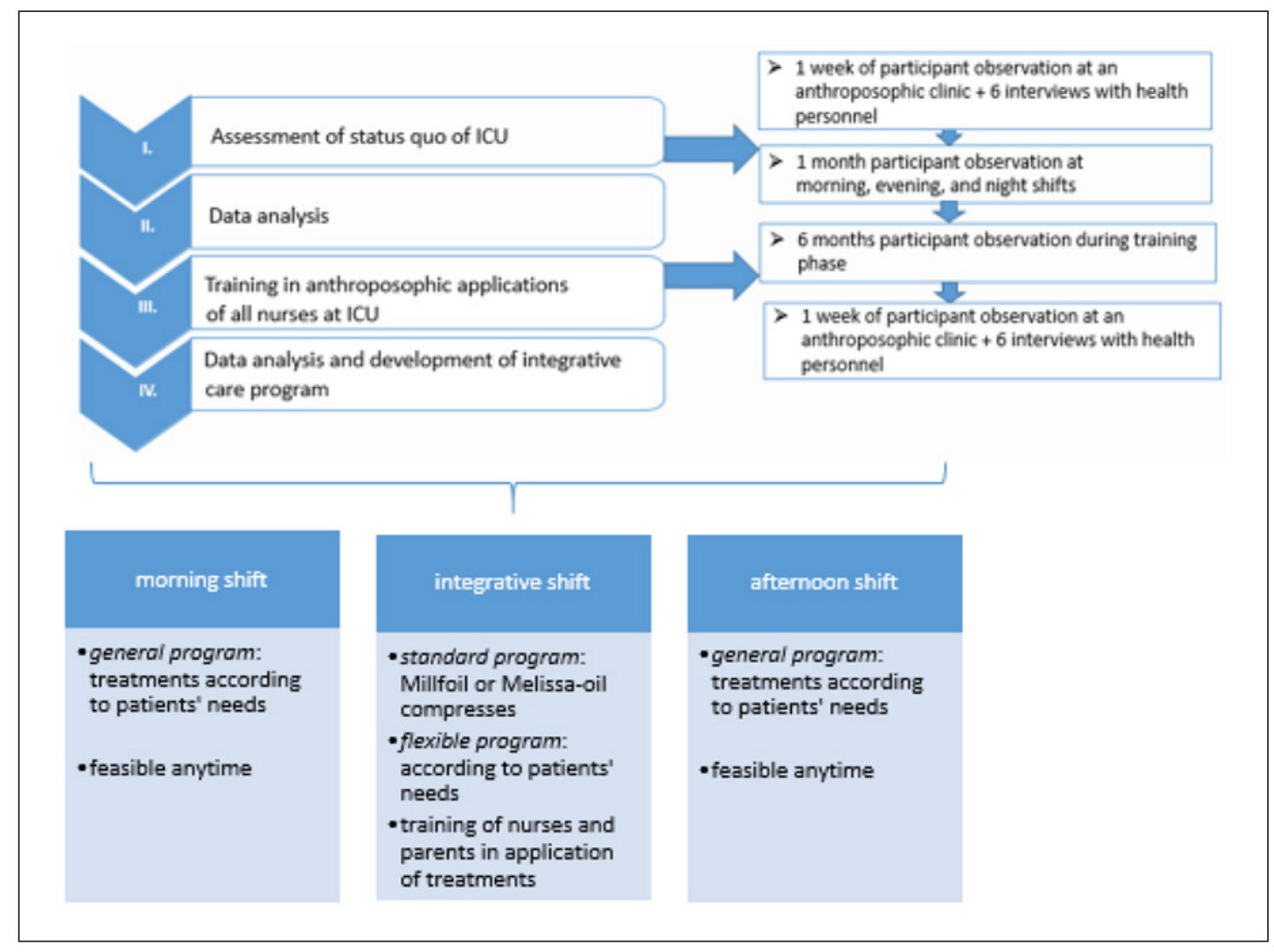

Fig. 1. Development of an integrative care program.

instruct new nursing staff members in the preparation and application of oil compresses and rhythmic embrocation. Finally, each treatment will be documented in the patients' charts (and in additional documentation for research purposes). The treatments offered will consist of a standard and flexible program:

Standard Program. A regular and reliable offer of integrative care is necessary for visibility and availability of the treatments for nurses, parents, and patients. The preceding training phase showed that oil compresses are easier to apply than rhythmic embrocation. Therefore, we chose 2 oil compresses as standard program for the integrative shift: Milfoil oil compress for nausea and vomiting and Melissa oil compress for anxiety and restlessness. Nausea and anxiety were previously identified as 2 of the most adverse side effects of conventional therapy and chemotherapy from which many of the young patients in the ICU suffer.

Flexible Program. Apart from the standard program, more flexible treatments, such as rhythmic embrocation of back, belly, or feet, as well as oil compresses will be offered in line with the needs of the patients and the indications. This flexible program will be provided on the same days as the standard program. The indications for treatments of the patients will be identified during the hando- ver from the morning to the afternoon shift and in consultation with the patients and their parents.

General Program. In addition to the standard and flexible programs offered in the integrative shift, anthroposophic treatments should be available during every shift, depending on the nurses' time and the indications and needs of the patient. Therefore, all nurses of the ward were trained in the treatments during the training phase. Every new nursing staff member will receive training by the integrative nurses. Integrative care can thus be delegated from the integrative shift to the other nurses and shifts. This snowball system will enable a slow but successful full integration of integrative care on the ICU, beyond the actual implementation program time. Over time, every nurse on the ward will be trained in the treatments. The program development process is shown in Figure 1.

\section{Discussion}

This paper describes the development of an integrative care program. Results showed that the structure of the ward, communication and information, and time constraints are important factors that need to be considered 
in the development process of the program. The program yields a unique and new approach to integrative health care in the German university landscape, where very few similar projects are being implemented to date. One exception in the realm of pediatric oncology is Witten/Herdecke University in Germany, where in 2012 the Institute of Integrative Medicine (IfIM) was founded with the intention of focusing on and researching "Integrative and Personalized Health Care.” The situation in Witten/Herdecke, in contrast to the setting described here, already provides a pre-structured situation where T\&CM modalities have been implemented and applied for many years. The situation looks similar in the USA, where integrative care has been on the rise over the past decades and has become an integral part of many cancer centers across the USA $[16,17]$. In the Scandinavian countries and the Netherlands, the integration of T\&CM modalities into primary care has also developed substantially [31-33]. In Germany, integrative care is well established in anthroposophic clinics, which have a long history of IM [22]. Anthroposophic clinics in Germany also have a special agreement with the National Health Insurance system for funding of IM treatment [34]. The health care system still struggles to accept those T\&CM/CIM modalities that lack evidence-based support [35]. Accordingly, T\&CM/CIM research and professorships in IM are still dependent on third-party funding [19]. Therefore, the project described here is exceptional. Its aim is to develop a sustainable, substantial program, which may serve as a role model for other similar clinical settings. Nevertheless, like every program with entrepreneurial character, this study also has limitations.

\section{Limitations of the Study}

Firstly, the program has been established in a very particular setting with very specific structures that may not be similar in other clinical settings. The many changes and rotations as well as the high workload in the ICU may not be comparable to other settings, especially when not in a university hospital setting. Therefore, to understand different settings, comparative data were generated at anthroposophic hospitals where integrative care was part of the clinic setting. This gave us an understanding of the different cultures and philosophies of conventional care in an ICU of a university hospital as compared to integrative care in an anthroposophic hospital. Hence, the second limitation is that the philosophies and cultures of the existing conventional care program and the newly developed care program are important for a seamless acceptance of an integrative care program. Merging these cultures and philosophies is a process that takes time. The training program already revealed that time is essential for integrative care and is not always sufficiently available in the ICU. Not only did nurses believe that they did not have sufficient time, but they also initially did not accept the treatments, even though they had agreed to integrate anthroposophic treatments into daily care. This can be interpreted, among other things, as resistance to the different philosophies of conventional and integrative care. Witt et al. [25] suggest using the principle of mergers from corporate culture as a guideline for overcoming such cultural differences. In this process, the formation of an "integration team" [36] or a "visible champion," a key person on the ward that can be a role model for other staff, is helpful to internalize the visions and goals of a project. In this program, the 2 training (expert) nurses could be viewed as "visible champions." The nurses, however, were not part of the ICU. Therefore, 5 nurses of the ICU were chosen to be the "visible champions" to make integrative care interactively visible, communicable, and available at all times. During the training phase, communication about the treatment among the nurses as well as between patients, parents, and nurses also generated acceptance in the nursing team. Without the "visible champions," anthroposophic treatment would potentially be negated by the fact that nurses feel "we don't have time." Therefore, apart from the strong focus on factors like time, structure, and communication and interaction, "visible champions" should be an integral part of the development of an integrative care program. Another limitation of this specific project may also lie in the fact that it was a third-party funded project, with funding for staff members (e.g., the expert nurses as well as a nurse to supplement the time of the 5 specifically trained nurses who implement the integrative shift) and a research team, which also coordinated the project. This funding enabled program implementation that may not be possible in other pediatric oncology contexts. The next steps are, therefore, first, the implementation of the described care program and, second, the development of a wider applicable program available to other wards that do not have thirdparty funding.

\section{Implications for Further Research}

In order to be able to transfer the developed program to other clinical contexts, future studies should address: (1) the applicability and modification of the program to other clinical settings; (2) evaluation of the impact of integrative care on the philosophy and culture of a ward; (3) the impact of a team on the implementation of integrative care; (4) the effects of anthroposophic treatments on time, touch, and affection amongst nurses and patients [7-37]; and (5) the financial requirements to implement such a program within the structures of the health insurance system. Furthermore, the article describes the development of the program of integrative care, but it does not examine the implementation of this program, which will be the next step of the overall project. The documentation 
(qualitative and quantitative) and analysis of the implementation phase helps to understand the feasibility of the program, its benefits and disadvantages for the ICU and for pediatric health care. For this, further interdisciplinary collaboration (between nurses, medical practitioners, experts in T\&CM/CIM, and social scientists) is needed to conduct further studies. Research in such a complex environment as a pediatric oncology ICU calls for a complex data generation process, which comprises a mixture of qualitative, quantitative, process- and outcome-oriented, efficacy and application-oriented research methods [21]. Developing and implementing a program in such a setting may be called a complex intervention in complex systems $[38,39]$. Evaluating such a complex intervention is difficult, as described by Craig et al. [40], especially with the limitations that come with classical randomized controlled trials in settings where well-being and effects are not measurable in numbers [41]. The complexity of holistic, individualistic, and inductive [14] interventions in $\mathrm{T} \& \mathrm{CM}$ and integrative care and their potential synergistic effect requires innovative evaluative approaches. Model validity, which includes the need for research to address the unique therapeutic context of the intervention, is central to this kind of research. Classical randomized controlled trials are limited in their ability to address this need. Therefore, for further research in the realm of T\&CM/CIM and conventional medicine, a mixed methods approach that includes a range of relevant and holistic outcome measures is needed [42] and will be applied in the next steps of the 3-year project as described by Stritter et al. [21].

\section{Conclusion}

We conducted qualitative research in order to develop, integrate, and evaluate an integrative care program in the complex setting of a pediatric oncology ICU of a German university hospital. We brought together literature analyses and methodological considerations on program development in a setting of conventional care and described the procedural character of the study. In doing so, we demonstrated that it takes time, a willing team, adaptation to the structures of the ICU, and regular and ongoing training and communication. Constraints may come from the substantial time pressure in the labor-intensive ICU, and with it the reluctance of the nurses to really integrate something new into an already extremely tight work schedule. The program should contribute to more comprehensive and effective patient care and stimulate greater integration of time and touch in the pediatric oncology ICU. The next step is to implement the concept and evaluate its applicability and economic sustainability.

\section{Acknowledgements}

We thank Britta Wilde and Doris Rapp from the Gemeinschaftskrankenhaus Havelhöhe for their substantive contribution to the program development. We also thank Maja Messlin, Kerstin Hürst, Deborah Tepper, Kira Bindewald, and Corinna Golde for their contribution as integrative nurses on the ward. Further, we thank the ICU 30i at the Charité - Universitätsmedizin Berlin for making the study possible.

\section{Statement of Ethics}

Ethical approval was received from the ethics board of Charité - Universitätsmedizin Berlin (EA2/132/16). Written consent was obtained from all individual participants included in the study.

\section{Conflict of Interest Statement}

The authors have no conflicts of interest to declare.

\section{Funding Sources}

We thank the MAHLE Foundation (Stuttgart, Germany) and the Software AG Foundation (Darmstadt, Germany) for financing the study.

\section{Author Contributions}

B.R., W.S., and U.A. carried out the research. W.S., G.S., and C.H. contributed to the design of the care program. W.S., C.H., and G.S. reviewed and corrected the manuscript; B.R. prepared the final draft of the manuscript. All authors approved the final manuscript.

\section{References}

1 Saletta F, Seng MS, Lau LM. Advances in paediatric cancer treatment. Transl Pediatr. 2014 Apr;3(2):156-82.

2 Bryant R. Managing side effects of childhood cancer treatment. J Pediatr Nurs. 2003 Apr; 18(2):113-25.

3 Coulter I, Willis E. Explaining the growth of complementary and alternative medicine. Health Sociol Rev. 2007;16(3-4):214-25.
4 Joos S, Musselmann B, Miksch A, Rosemann $\mathrm{T}$, Szecsenyi J. The role of complementary and alternative medicine (CAM) in Germany - a focus group study of GPs. BMC Health Serv Res. 2008 Jun;8(1):127.

5 Laengler A, Spix C, Seifert G, Gottschling S, Graf N, Kaatsch P. Complementary and alternative treatment methods in children with cancer: A population-based retrospec- tive survey on the prevalence of use in Germany. Eur J Cancer. 2008 Oct;44(15):223340.

6 Längler A, Mansky PJ, Seifert G, editors. Integrative Pediatric Oncology. Berlin, Heidelberg, New York, Barcelona, Hong Kong, London, Milan, Paris, Singapore, Tokyo: Springer; 2012. https://doi.org/10.1007/978-3-64204201-0. 
7 Magi T, Kuehni CE, Torchetti L, Wengenroth L, Lüer S, Frei-Erb M. Use of Complementary and Alternative Medicine in Children with Cancer: A Study at a Swiss University Hospital. PLoS One. 2015 Dec;10(12):e0145787.

8 Eisenberg DM, Davis RB, Ettner SL, Appel S, Wilkey S, Van Rompay M, et al. Trends in alternative medicine use in the United States, 1990-1997: results of a follow-up national survey. JAMA. 1998 Nov;280(18):1569-75.

9 Boon HS, Kachan N. Integrative medicine: a tale of two clinics. BMC Complement Altern Med. 2008 Jun;8(1):32.

10 Boon H, Verhoef M, O’Hara D, Findlay B, Majid N. Integrative healthcare: arriving at a working definition. Altern Ther Health Med. 2004 Sep-Oct;10(5):48-56.

11 Holmberg C, Brinkhaus B, Witt C. Experts' opinions on terminology for complementary and integrative medicine - a qualitative study with leading experts. BMC Complement $\mathrm{Al}$ tern Med. 2012 Nov;12(1):218.

12 McFadden KL, Hernández TD, Ito TA. Attitudes toward complementary and alternative medicine influence its use. Explore (NY). 2010 Nov-Dec;6(6):380-8.

13 Dobos G. Integrative medicine - medicine of the future or 'old wine in new skins'?. Eur J Integr Med. 2009;1(3):109-15.

14 Barrett B, Marchand L, Scheder J, Plane MB, Maberry R, Appelbaum D, et al. Themes of holism, empowerment, access, and legitimacy define complementary, alternative, and integrative medicine in relation to conventional biomedicine. J Altern Complement Med. 2003 Dec;9(6):937-47.

15 Frass M, Strassl RP, Friehs H, Müllner M, Kundi M, Kaye AD. Use and acceptance of complementary and alternative medicine among the general population and medical personnel: a systematic review. Ochsner J. 2012;12(1):45-56.

16 Cassileth BR. The Integrative Medicine Service at Memorial Sloan-Kettering Cancer Center. Semin Oncol. 2002 Dec;29(6):585-8.

17 Horrigan B, Lewis S, Abrams DI, Pechura C. Integrative Medicine in America-How Integrative Medicine Is Being Practiced in Clinical Centers Across the United States. Glob Adv Health Med. 2012;1(3):18-94.

18 Willich SN, Girke M, Hoppe JD, Kiene H, Klitzsch W, Matthiessen PF, et al. Schulmedizin und Komplementärmedizin: Verständnis und Zusammenarbeit müssen vertieft werden. Dtsch Arztebl. 2004;101(19):A-1314.
19 Albrecht H. Zur Lage der Komplementärmedizin in Deutschland. Complement Med Res. 2013;20(1):73-7.

20 Pérard M, Mittring N, Schweiger D, Kummer C, Witt CM. Merging conventional and complementary medicine in a clinic department - a theoretical model and practical recommendations. BMC Complement Altern Med. 2015 Jun;15(1):172.

21 Stritter W, Rutert B, Längler A, Eggert A, Holmberg C, Seifert G. Integrative care for children with cancer. Project design for the development of an integrative care programme for use in paediatric oncology. Complement Ther Med. 2018 Dec;41:247-51.

22 Kienle GS, Albonico HU, Baars E, Hamre HJ, Zimmermann P, Kiene H. Anthroposophic medicine: an integrative medical system originating in Europe. Glob Adv Health Med. 2013 Nov;2(6):20-31.

23 Wälchli C, Saltzwedel G, Krüerke D, Kaufmann C, Schnorr B, Rist L, et al. Physiologic effects of rhythmical massage: a prospective exploratory cohort study. J Altern Complement Med. 2014 Jun;20(6):507-15.

24 Post-White J, Fitzgerald M, Hageness S, Sencer SF. Complementary and alternative medicine use in children with cancer and general and specialty pediatrics. J Pediatr Oncol Nurs. 2009;26(1):7-15

25 Witt CM, Pérard M, Berman B, Berman S, Birdsall TC, Defren H, et al. Using the framework of corporate culture in "mergers" to support the development of a cultural basis for integrative medicine - guidance for building an integrative medicine department or service. Patient Prefer Adherence. 2015 Jan;9: 113-20.

26 Ranheim A, Kärner A, Arman M, Rehnsfeldt AW, Berterö C. Embodied reflection in practice - 'touching the core of caring'. Int J Nurs Pract. 2010 Jun;16(3):241-7.

27 Sülz S, Langhammer K, Becker-Peth M, Roth B. What drives perceived work intensity in neonatal intensive care units? Empirical evidence from a longitudinal study. J Adv Nurs. 2017 Oct;73(10):2441-9.

28 Charmaz K. Constructing Grounded Therapy: A Practical Guide through Qualitative Analysis. London: Sage Publications; 2014.

29 Glaser BG, Strauss AL. The discovery of grounded theory: strategies for qualitative research. London: Transaction Publishers; 1999.
30 Noble H, Smith J. Issues of validity and reliability in qualitative research. Evid Based Nurs. 2015 Apr;18(2):34-5.

31 Sundberg T, Halpin J, Warenmark A, Falkenberg T. Towards a model for integrative medicine in Swedish primary care. BMC Health Serv Res. 2007 Jul;7(1):107.

32 Jong MC, van Vliet M, Huttenhuis S, van der Veer D, van den Heijkant S. Attitudes toward integrative paediatrics: a national survey among youth health are physicians in The Netherlands. BMC Complement Altern Med. 2012 Jan;12(1):4.

33 von Rosenstiel IA, Schats W, Bongers K, Jong MC. Integrative paediatrics: a Dutch experience. Focus Altern Complement Ther. 2011; 16(1):22-7.

34 Jansen E. The Role of Complementary and Alternative Medicine in the Healthcare System: A German Paradox. Complement Med Res. 2017;24(5):290-4.

35 Ernst E. How Much of CAM Is Based on Research Evidence?. Evid Based Complement Alternat Med. 2011;2011:676490.

36 Tetenbaum TJ. Beating the Odds of Merger \& Acquisition Failure: Seven Key Practices That Improve the Chance for Expected Integration and Synergies. Organ Dyn. 1999;28(2):22-36.

37 Edvardsson JD, Sandman PO, Rasmussen $\mathrm{BH}$. Meanings of giving touch in the care of older patients: becoming a valuable person and professional. J Clin Nurs. 2003 Jul;12(4): 601-9.

38 Hunter DJ. Role of politics in understanding complex, messy health systems: an essay by David J Hunter. BMJ. 2015 Mar;350:h1214.

39 Shiell A, Hawe P, Gold L. Complex interventions or complex systems? Implications for health economic evaluation. BMJ. 2008 Jun; 336(7656):1281-3.

40 Craig P, Dieppe P, Macintyre S, Michie S, Nazareth I, Petticrew M; Medical Research Council Guidance. Developing and evaluating complex interventions: the new Medical Research Council guidance. BMJ. 2008 Sep; 337:a1655.

41 Leis AM, Weeks LC, Verhoef MJ. Principles to guide integrative oncology and the development of an evidence base. Curr Oncol. 2008 Aug;15(0 Suppl 2):s83-7.

42 Verhoef MJ, Lewith G, Ritenbaugh C, Boon $\mathrm{H}$, Fleishman S, Leis A. Complementary and alternative medicine whole systems research: beyond identification of inadequacies of the RCT. Complement Ther Med. 2005 Sep; 13(3):206-12. 